\section{Food safety: correct information for pregnant women}

\author{
Bartolomeo Griglio, ${ }^{1}$ Daniele Nucera, ${ }^{2}$ \\ Federica Vergano, ${ }^{3}$ Maria Ausilia Grassi ${ }^{2}$
}

'ASL TO5, Torino, Italy; ${ }^{2}$ Dipartimento di Patologia Animale, Università degli Studi di Torino, Grugliasco, Italy; ${ }^{3}$ Tecnico della Prevenzione, Torino, Italy

\section{Abstract}

This study was aimed at investigating the knowledge of pregnant women on food safety with particular attention to the effectiveness of the informative material (pamphlet and poster) prepared in a previous study. To this scope, a questionnaire composed by 8 questions (Likert scaled) was used except for one which was a Y/N question. Themes of the questionnaire were: level of concerns on food safety, and knowledge on foodborne diseases (salomonellosis, toxoplasmosis and listeriosis), risk factors and preventive measures. Results indicate that knowledge increased in respect to that of the previous study, but in relation to informative material previously distributed.

Nella discussione sulla sicurezza alimentare appare fondamentale il concetto di rischio che può essere ricondotto ad ogni situazione che potrebbe rappresentare una minaccia per $\mathrm{i}$ consumatori. L'analisi del rischio risulta lo strumento più efficace al fine di identificare $\mathrm{i}$ potenziali effetti avversi per la salute pubblica da parte dei microrganismi patogeni che contaminano gli alimenti (risk assessment) e per la formulazione delle ipotesi relative alla gestione dei rischi (risk management) (Frewer et al, 1998). Un altro aspetto significativo è sicuramente la percezione del rischio, intesa come l'entità delle conseguenze del danno prodotte da un determinato comportamento. Questa percezione introduce, altresì, la dimensione della fiducia, termine declinato più efficacemente con i termini inglesi confidence e trust, (Belliggiano, 2009), che si riferiscono, rispettivamente, ad una fiducia passiva conseguente all'incapacità di agire sugli eventi e ad una fiducia attiva, subordinata invece al controllo consapevole degli stessi. Appare quindi evidente l'importanza della risk communication rivolta in particolare, in linea con quanto stabilito dall'OMS, ai cosiddetti High-RiskGroups, rappresentati da donne in gravidanza, bambini, anziani ed immunodepressi. A distanza di 5-6 anni da una precedente indagine condotta presso l'ASL T05, si è voluto valutare il livello di informazione delle donne in gravidanza rispetto ai rischi connessi all'alimentazione e, in particolare, valutare l'efficacia dei presidi informativi, opuscolo e poster, scaturiti dai risultati dell'indagine precedente. È stato quindi allestito un questionario costituito da 8 domande a scale numeriche asimmetriche a sette modalità (scala Linkert) a risposta chiusa per lo più di tipo dicotomico e polifonico (variabili score) e da una domanda avente come risposte SI e NO (variabili binarie), finalizzate ad indagare: i) il livello di preoccupazione delle donne rispetto alle tematiche inerenti la sicurezza alimentare in gravidanza; ii) il grado di conoscenza dei fattori di rischio e delle modalità di prevenzione relativamente a salmonellosi, toxoplasmosi, stafilococcosi e listeriosi, a seguito della redazione degli strumenti informativi messi a punti al termine del progetto precedente.

Il questionario è stato somministrato alle donne al settimo mese che frequentavano i corsi pre-parto presso le sedi dei corsi dislocate su tutto il territorio dell'ASL T05 (Consultorio Familiare Debouché di Nichelino, Ospedale San Lorenzo di Carmagnola, Ospedale Santa Croce di Moncalieri) (campione $1, \mathrm{~N}=100$ ) ed alle gestanti ancora al primo trimestre di gravidanza (campione 2, $\mathrm{N}=44$ ) presso l'Ospedale Maggiore di Chieri. Dei 100 questionari somministrati al campione 1 ne sono stati considerati ed analizzati 95: sono stati esclusi dallo studio quelli che presentavano un numero di missing troppo elevato o che avevano risposte polarizzate solo su alcuni valori delle scale Likert (solo il valore minimo e/o solo il valore massimo). I questionari distribuiti alle gestanti al primo trimestre sono stati esaminati nella loro totalità $(\mathrm{N}=44)$. L'analisi statistica è stata condotta: i) sulle variabili score, volta al confronto tra gli score attribuiti dalle donne che avevano compilato il questionario durante la frequenza al corso preparto con quelli attribuiti dalle donne che lo avevano compilato durante il primo trimestre di gravidanza. L'analisi è stata eseguita con il test di Mann-Withney, utilizzando il software $\mathrm{SAS}^{\circledR}$, considerando significativi i risultati con $\mathrm{P}<0,05$; ii) sulle variabili binarie. Per ognuna delle due variabili è stata costruita una tabella $2 \times 2$ (righe: i due gruppi di appartenenza del campione, partecipanti al corso pre-parto primo trimestre di gravidanza; colonne: le due possibili risposte alla domanda $(1=s i, 0=n 0)$. Il calcolo delle frequenze è stato eseguito utilizzando il $\chi^{2}$ (significatività se $\mathrm{P}<0,05$ ).

Il campione 1 è risultato costituito da 95 donne, di età media 31 anni (range compreso tra 19 e 41 anni); il 91\% è risultato alla prima gravidanza a termine, mentre il $9 \%$ ad una gravidanza successiva. Il $60 \%$ del campione ha conseguito il diploma di scuola superiore ed il $38 \%$ svolge la professione di impiegata.

Il campione 2 è risultato composto da 44 gestanti, di età media 33 anni (range compre-
Correspondence: Maria Ausilia Grassi, Dipartimento di Patologia Animale, Università degli Studi di Torino, via Leonardo da Vinci 48, 10095 Grugliasco, Italy.

Tel: +39.011.6709218 - Fax: +39.011.6709224.

E-mail: auxilia.grassi@unito.it

Key words: Pregnancy, Food Safety, Risk communication, Questionnaire.

Conflict of interests: the authors declare no potential conflict of interests.

Received for publication: 15 January 2013.

Revision received: 21 January 2013.

Accepted for publication: 22 January 2013.

This work is licensed under a Creative Commons Attribution 3.0 License (by-nc 3.0).

(C) Copyright B. Griglio et al., 2013

Licensee PAGEPress, Italy

Italian Journal of Food Safety 2013; 2:el doi:10.4081/ijfs.2013.e1

so tra 20 e 42 anni); il 50\% del campione affrontava la prima gravidanza mentre il restante $50 \%$ una gravidanza successiva. Il $45 \%$ del campione dichiara di aver conseguito il diploma di scuola superiore ed il 25\% svolge il lavoro di impiegata.

Alla domanda 1 Quanto si ritiene informata sui rischi legati al consumo degli alimenti? la frequenza delle donne che si ritengono informate non è risultata statisticamente differente quando si sono confrontate le risposte date dalle donne del campione 2 (59\%) con quelle delle gestanti intervistate nella prima indagine (44\%), mentre una differenza significativa è stata osservata nel confronto tra le donne a fine gravidanza del campione 1 (83\%) e quelle del primo progetto (44\%). Risulta pertanto evidente un netto miglioramento del livello di informazione delle gestanti (cambiamento statisticamente significativo). Inoltre, la percentuale maggiore di donne informate appartiene al campione 1 ( $83 \%$ rispetto al $59 \%$ del campione 2), facendo quindi supporre che le informazioni utili vengano fornite prevalentemente in una fase avanzata della gravidanza, quando le azioni preventive potrebbero non essere più così efficaci per un significativo controllo del rischio.

La domanda 2 Da quali di queste fonti ha avuto informazioni sui rischi legati agli alimenti? ha evidenziato come entrambi i campioni di donne diano maggior credito all'agenda della gravidanza (distribuita all'ottava settimana di gravidanza): 93,68\% (campione 1) e $79,55 \%$ (campione 2). Particolare importanza viene data al ruolo delle ostetriche (campione $1=88,42 \%$ e campione $2=72,73 \%$ ), ma anche alle conversazioni con amici, conoscenti e negozianti di fiducia (campione $1=84,21 \%$ e 
campione $2=79,55 \%$ ) ed infine i programmi o articoli dei mass media (campione $1=62,11 \%$ e campione $2=68,18 \%$ ).

Per quanto riguarda la domanda 3 Quanto ritiene rischioso per la salute della donna in gravidanza ciascuno dei seguenti aspetti? i due campioni di donne hanno evidenziato un livello di sensibilità quasi del tutto sovrapponibile ai rischi correlati al consumo eccessivo di alcool, sale, caffeina, calorie e alimenti contaminati da microrganismi (batteri, virus, ecc.).

Alla domanda 4 Quanto ritiene rischioso per la salute del bambino durante la gravidanza ciascuno dei seguenti aspetti? entrambi i campioni hanno indicato come rischio maggiore $\mathrm{i}$ microrganismi (per entrambi i campioni il $6,9 \%)$ - risposta verosimilmente influenzata dall'evento batterio killer - e l'alcool (rispettivamente: $6,9 \%$ per il campione 1 e $6,7 \%$ per il campione 2).

La domanda 5 Secondo lei, quanto ciascuna delle seguenti malattie può influire sulla salute della donna in gravidanza? ha avuto lo stesso tipo di risposta, pur se con percentuali differenti, dai due campioni ed in particolare il ruolo di maggior responsabile è stato attribuito a toxoplasma, seguito da salmonella, stafilococco ed infine listeria. La stessa distribuzione si è osservata in risposta alla domanda 6 Secondo lei, quanto ciascuna delle seguenti malattie può influire sulla salute del bambino durante la gravidanza? Infine, in merito alla sicurezza degli alimenti in gravidanza (domande 7 e 8), per entrambi i campioni, frutta e verdura sono considerati più sicuri mentre i prodotti carnei sono quelli reputati più a rischio. Infine, relativamente alle misure preventive (domanda 9), entrambi i campioni hanno attribuito maggior importanza alla cottura (campione 1 e 2, rispettivamente: 6,7\% e $6,8 \%)$ e all'utilizzo di materie prime sicure (campione 1 e 2, rispettivamente: 6,4\% e $6,5 \%)$.

Il confronto dei risultati ottenuti dal primo progetto e da quelli relativi al campione attuale delle donne partecipanti al corso pre-parto ha evidenziato un maggior livello di conoscenze ed informazioni. Dalle percentuali ottenute in relazione alla lettura dell'opuscolo, si può affermare che la sua distribuzione non sia stata sufficientemente diffusa; infatti, i mezzi di comunicazione a cui entrambe le categorie di gestanti (pre-parto e primo trimestre) hanno dato maggiore credito sono stati l'agenda della gravidanza ed i colloqui con le ostetriche. Il poster, invece, non è stato riscontrato in nessuno dei luoghi in cui è avvenuta la somministrazione dei questionari.

L'analisi invece dei risultati emersi dai due campioni della presente ricerca evidenzia un maggior livello d'informazione nel gruppo di donne frequentanti il corso pre-parto, nonostante la distribuzione del materiale informativo avvenga a partire solo dall'ottava settimana di gestazione.

\section{Bibliografia}

Belliggiano A, 2009. Percezione della sicurezza alimentare e nuovi modelli di organizzazione della produzione. Rivista di diritto alimentare 3:1-6. Disponibile al sito: http://www.rivistadirittoalimentare.it/rivista/2009-04/BELLIGGIANO.pdf Ultimo accesso: 21/01/2013.

Frewer LJ, Howard C, Hedderley D, Shepherd $\mathrm{R}$, 1998. Methodological approaches to assessing risk perceptions associated with food-related hazards. Risk Anal 18:95-102. 